Veröffentlichungen des Forschungsinstituts für vergleichende

Religionsgeschichte an der Universität Leipzig

\title{
Bibliographie
}

zur Frage

nach den Wechselbeziehungen zwischen

\section{Buddhismus und Christentum}

\author{
Von
}

Dr. Hans Haas

Profossor der Religionsgeschichto

an der Universitat Loipzig

Leipzig

J. C. Hinrichs'sche Buchhandlung 1922 


\section{VERÖFFENTLICHUNGEN \\ DES STAATLICHEN FORSCHUNGSINSTITUTS FÜR VERGLEICHENDE RELIGIONSGESCHICHTE \\ AN DER UNIVERSITÄT LEIPZIG}

HERAUSGEGEBEN VON PROF. DR. HANS HAAS

Nr. 1. Religionsgeschichtliche Bibliographie Jahrgang I/II, III/IV, V/VI, die Literatur der Jahre 1914 und 1915, bzw. 1916 und 1917 sowie 1918 und 1919 enthaltend.

(Wird fortgesetzt)

Nr. 2. Allgemeine Religionskunde in Bildern. Probelieferung: Die Buddha-Legende auf den Flachreliefs

- der ersten Galerie des Stupa von Boro-Budur (Java).

(Erklärender Text in Vorbereitung)

Nr. 3. Heińrich Frick, Ghazälīs Selbstbiographie. Ein Vergleich mit Augustirs Konfessionen.

Nr. 4. Hans-Leisegang, Pneuria hagion. Der Ursprung des Geistbegriffs der synoptischen Evangelien aus der griechischen Mystik.

Nr. 5. Hans Haas, "Das Scherflein der Witwe" und seine Entsprechung im Tripițaka.

Nr. 6. Hans Haas, Bibliographie zur Frage nach den Wechselbeziehungen zwischen Buddhismus und Christentum.

(Nr. 6 als Anhang auch in Nr. 5 enthalten)

Nr. 1 im Verlag, bzw. Kommissionsverlag von B. G. Teubner, Leipzig.

Nr. 3-6 im Verlag der J. C. Hinrichs'schen Buchhandlung, Leipzig. 


\title{
B IBLIOGR A PHIE
}

ZUR FRAGE

NACH DEN WECHSELBEZIEHUNGEN ZWISCHEN

\section{BUDDHISMUS UND CHRISTENTUM}

\author{
VON \\ HANS HAAS
}

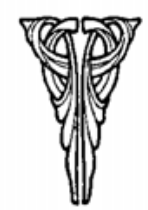


